\title{
Nonautonomous transcritical and pitchfork bifurcations in impulsive systems
}

\author{
M. U. Akhmet and A. Kashkynbayev
}




\title{
NONAUTONOMOUS TRANSCRITICAL AND PITCHFORK BIFURCATIONS IN IMPULSIVE SYSTEMS
}

\author{
M. U. AKHMET AND A. KASHKYNBAYEV
}

Received 3 September, 2013

\begin{abstract}
For the first time analogues of nonautonomous transcritical and pitchfork bifurcations are investigated for impulsive systems.

2010 Mathematics Subject Classification: 34A37; 34C23; 34D05; 37B55; 37G35; 34D45; 37C70; 37C75

Keywords: nonautonomous bifurcation theory, impulsive differential equations, attractive solution, repulsive solution, Pitchfork bifurcation, transcritical bifurcation
\end{abstract}

\section{INTRODUCTION}

Investigation of states of time dependent dynamical systems leads to nonautonomous problems in the form of the equations of perturbed motions. If the model depends on parameters, it is the main object of nonautonomous bifurcation theory to describe the qualitative changes when these parameters are varied. Extending nonautonomous bifurcation theory to impulsive systems is a contemporary problem. In continuous dynamical systems, this theory is studied in [10,11,13,14,16,17].

Many evolutionary processes in the real world are characterized by sudden changes at certain times. These changes are called impulsive phenomena $[4,5,12,18,19]$, which are widespread in modeling of mechanics, electronics, biology, neural networks, medicine, and social sciences [1,2,8,15,18-21]. An impulsive differential equation is one of the basic instruments to understand better the role of discontinuity in the real world problems. There are qualitative studies on asymptotic properties of the quasilinear impulsive differential equations and ordinary differential equations $[3-6,9,12,19]$. The main novelty of this paper is the study of the analogues of nonautonomous pitchfork and transcritical bifurcations in scalar impulsive systems, in which the properties of the system depend on the entire time, both of past and future.

The second author was supported in part by the TUBITAK, the Scientific and Technological Research Council of Turkey, BIDEB 2215 Program. 
This paper is organized as follows. In Section 2 we give basic definitions and definitions of all-time, past and future attractors. In Section 3 results on linearized attractivity and repulsivity are presented. Section 4 and Section 5 is devoted to impulsive analogues of nonautonomous transcritical and pitchfork bifurcations, respectively.

\section{Preliminaries}

We denote by $\mathbb{R}$ the set of all real numbers, by $\mathbb{Z}$ the set of integers and write $\mathbb{R}_{k}^{-}:=$ $[-\infty, k)$ and $\mathbb{R}_{k}^{+}:=[k, \infty)$ for given $k \in \mathbb{R}$. In this section we introduce the concepts of attractive and repulsive solutions, which are used to analyze asymptotic behavior of impulsive non-autonomous systems. This paper is concerned with systems of the type

$$
\begin{aligned}
& \dot{x}=f(t, x), \\
& \left.\Delta x\right|_{t=\theta_{i}}=J_{i}(x),
\end{aligned}
$$

where $\left.\Delta x\right|_{t=\theta_{i}}:=x\left(\theta_{i}+\right)-x\left(\theta_{i}\right), x\left(\theta_{i}+\right)=\lim _{t \rightarrow \theta_{i}^{+}} x(t)$. The system (2.1) is defined on the set $\Omega=I \times \mathbb{A} \times G$ where $G \subseteq \mathbb{R}^{n}, I$ is the interval of the form $I=\mathbb{R}$, $I=\mathbb{R}_{k}^{-}$or $I=\mathbb{R}_{k}^{+}$, respectively. $\theta$ is a nonempty sequence with the set of indexes A such that $\left|\theta_{i}\right| \rightarrow \infty$ as $|i| \rightarrow \infty$. Let $\phi\left(t, t_{0}, x_{0}\right)$ be solution of (2.1) which is continuable and unique on $I$.

Denote by $P C(\mathbb{R}, \theta)$ the space of piecewise left continuous functions with discontinuity of the first kind at points in $\theta$. The Euclidean space $\mathbb{R}^{n}$ is equipped with the norm $\|\cdot\|$, and write $B_{\epsilon}\left(x_{0}\right)=\left\{x \in \mathbb{R}^{n}:\left\|x-x_{0}\right\|<\epsilon\right\}$ for arbitrary $\epsilon$-neighborhood of some point $x_{0} \in \mathbb{R}^{n}$. We use Hausdorff semi-distance between nonempty set $A$ and $B$ as $d(A, B)=\sup _{a \in A} \inf _{b \in B} d(a, b)$. For arbitrary nonempty set $X \subset \mathbb{R}^{n}$ define $\phi\left(t, t_{0}, X\right):=\bigcup_{x_{0} \in X} \phi\left(t, t_{0}, x_{0}\right)$.

A set $M \subset I \times \mathbb{R}^{n}$ is called nanautonomous set if for all $t \in I$, the $t-$ fibers $M(t):=\left\{x \in \mathbb{R}^{n}:(t, x) \in M\right\}$ are nonempty. $M$ is said to be compact if all t-fibers are compact. $M$ is said to be invariant if $\phi\left(t, t_{0}, M\left(t_{0}\right)\right)=M(t)$ for all $t, t_{0} \in I$.

Asymptotic properties of continuous dynamics and dynamics with discontinuous are the same. In what follows we use definitions of attractivity and repulsivity without any changes form [17].

Definition 1. Let $I=\mathbb{R}, A$ and $R$ be compact and invariant nonautonomous sets and $\psi: \mathbb{R} \rightarrow \mathbb{R}^{n}$ be a solution of the system (2.1).

- $A$ is called all-time attractor if there exists an $\eta>0$ with

$$
\lim _{t \rightarrow \infty} \sup _{t_{0} \in \mathbb{R}} d\left(\phi\left(t+t_{0}, t_{0}, B_{\eta}\left(A\left(t_{0}\right)\right)\right), A\left(t+t_{0}\right)\right)=0 .
$$

The supremum of all positive $\eta$ with this property is called all-time attraction radius of $A$ and denoted by $\mathcal{A}_{A}^{ \pm}$.

- $\psi$ is called all-time attractive if graph $\psi$ is an all-time attractor. 
- $R$ is called all-time repeller if there exists an $\eta>0$ with

$$
\lim _{t \rightarrow \infty} \sup _{t_{0} \in \mathbb{R}} d\left(\phi\left(t_{0}-t, t_{0}, B_{\eta}\left(R\left(t_{0}\right)\right)\right), R\left(t_{0}-t\right)\right)=0 .
$$

The supremum of all positive $\eta$ with this property is called all-time repulsion radius of $R$ and denoted by $\mathcal{R}_{R}^{ \pm}$.

- $\psi$ is called all-time repulsive if graph $\psi$ is an all-time repeller.

In what follows we use past attractivity and repulsivity in the asymptotic analysis of the systems that depend on the past. Note that a past attractor is a local form of a pullback attractor [7].

Definition 2. Let $I=\mathbb{R}_{k}^{-}, A$ and $R$ be compact and invariant nonautonomous sets and $\psi: \mathbb{R} \rightarrow \mathbb{R}^{n}$ be a solution of the system (2.1).

- $A$ is called past attractor if there exists an $\eta>0$ with

$$
\lim _{t \rightarrow \infty} d\left(\phi\left(t_{0}, t_{0}-t, B_{\eta}\left(A\left(t_{0}-t\right)\right)\right), A\left(t_{0}\right)\right)=0 \text { for all } t_{0} \in I .
$$

The supremum of all positive $\eta$ with this property is called past attraction radius of $A$ and is denoted by $\mathcal{A}_{A}^{-}$.

- $\psi$ is called past attractive if the graph $\psi$ is a past attractor.

- $R$ is called past repeller if there exists an $\eta>0$ with

$$
\lim _{t \rightarrow \infty} d\left(\phi\left(t_{0}-t, t_{0}, B_{\eta}\left(R\left(t_{0}\right)\right)\right), R\left(t_{0}-t\right)\right)=0 \text { for all } t_{0} \in I .
$$

The supremum of all positive $\eta$ such that there exists a $\widehat{k} \in I$ with

$$
\lim _{t \rightarrow \infty} d\left(\phi\left(t_{0}-t, t_{0}, B_{\eta}\left(R\left(t_{0}\right)\right)\right), R\left(t_{0}-t\right)\right)=0 \text { for all } t_{0} \leq \widehat{k}
$$

is called past repulsion radius of $R$ and denoted by $\mathcal{R}_{R}^{-}$.

- $\psi$ is called past repulsive if graph $\psi$ is an past repeller.

Definition 3. Let $I=\mathbb{R}_{k}^{+}, A$ and $R$ be compact and invariant nonautonomous sets and $\psi: \mathbb{R} \rightarrow \mathbb{R}^{n}$ be a solution of the system (2.1).

- $A$ is called future attractor if there exists an $\eta>0$ with

$$
\lim _{t \rightarrow \infty} d\left(\phi\left(t+t_{0}, t_{0}, B_{\eta}\left(A\left(t_{0}\right)\right)\right), A\left(t+t_{0}\right)\right)=0 \text { for all } t_{0} \in I .
$$

The supremum of all positive $\eta$ such that there exists a $\widehat{k} \in I$ with

$$
\lim _{t \rightarrow \infty} d\left(\phi\left(t+t_{0}, t_{0}, B_{\eta}\left(A\left(t_{0}\right)\right)\right), A\left(t+t_{0}\right)\right)=0 \text { for all } t_{0} \geq \widehat{k}
$$

is called future attraction radius of $A$ and is denoted by $\mathcal{A}_{A}^{+}$.

- $\psi$ is called future attractive if graph $\psi$ is a future attractor. 
- $R$ is called future repeller if there exists an $\eta>0$ with

$$
\lim _{t \rightarrow \infty} d\left(\phi\left(t_{0}, t_{0}+t, B_{\eta}\left(R\left(t+t_{0}\right)\right)\right), R\left(t_{0}\right)\right)=0 \text { for all } t_{0} \in I .
$$

The supremum of all positive $\eta$ with this property is called future repulsion radius of $R$ and is denoted by $\mathcal{R}_{R}^{+}$.

- $\psi$ is called future repulsive if graph $\psi$ is a future repeller.

From the definitions given above it follows that every future attractive solution is uniformly asymptotically stable. Moreover, every all-time attractor (repeller, respectively) is both a past attractor (repeller, respectively) and a future attractor (repeller, respectively).

\section{LINEARIZED ATTRACTIVITY AND REPULSIVITY}

In this section we study linearized systems with definitions provided in the previous section which play great role in the stability analysis of solutions of nonlinear impulsive systems with fixed moments of impulses. We consider the system with interval $I$ of the form $\mathbb{R}, \mathbb{R}_{k}^{-}$or $\mathbb{R}_{k}^{+}$, respectively, and let

$$
\begin{aligned}
& \dot{x}=A(t) x+F(t, x), \\
& \left.\Delta x\right|_{t=\theta_{i}}=B_{i} x+I_{i}(x),
\end{aligned}
$$

where $A \in P C(I, \theta)$, matrices $B_{i}$ satisfy $\operatorname{det}\left(B_{i}+I\right) \neq 0, F: I \times G \rightarrow \mathbb{R}^{n}$ and $I: \mathbb{A} \times G \rightarrow \mathbb{R}^{n}$. An infinite sequence $\theta_{i}$ satisfies $\left|\theta_{i}\right| \rightarrow \infty$ as $|i| \rightarrow \infty$. It is assumed that there exist positive constants $\underline{\theta}$ and $\bar{\theta}$ such that $\underline{\theta} \leq \theta_{i+1}-\theta_{i} \leq \bar{\theta}$. Denote $\phi\left(t, t_{0}, x_{0}\right)$ the solution of (3.1) and let $\Phi(t, s)$ be the fundamental matrix of the linearized system

$$
\begin{aligned}
& \dot{x}=A(t) x, \\
& \left.\Delta x\right|_{t=\theta_{i}}=B_{i} x .
\end{aligned}
$$

A version of the next theorem for future time has been proved in [19]. We will extend this result for all-time attractiveness.

Theorem 1. If there exist $\beta<0, K \geq 1$ and $\delta>0$ such that

$$
\|\Phi(t, s)\| \leq K e^{\beta(t-s)} \text { for all } t \geq s,
$$

and the functions $F(t, x)$ and $I_{i}(x)$ satisfy the inequalities

$$
\|F(t, x)\| \leq l\|x\|, \quad\left\|I_{i}(x)\right\| \leq l\|x\|
$$

for all $t \in I, i \in \mathbb{A}$ and $\|x\|<h, h>0$. Then,

$$
\left\|\phi\left(t, t_{0}, x_{0}\right)\right\| \leq \delta e^{\left(\beta+K l+\frac{1}{\underline{\underline{\theta}}} \ln (1+K l)\right)\left(t-t_{0}\right)} \text { for all } t, t_{0} \in I \text { with } t \geq t_{0},
$$

i.e., for sufficiently small values of $l$, the trivial solution of (3.1) is all-time (past, future, respectively) attractive. 
Proof. An equivalent integral equation of the system (3.1) can be written as [4,19]:

$$
\begin{aligned}
\phi\left(t, t_{0}, x_{0}\right)=\Phi\left(t, t_{0}\right) x_{0}+\int_{t_{0}}^{t} \Phi(t, s) F\left(s, \phi\left(s, t_{0}, x_{0}\right)\right) d s & \\
& +\sum_{t_{0} \leq \theta_{i}<t} \Phi\left(t, \theta_{i}\right) I_{i}\left(\phi\left(\theta_{i}, t_{0}, x_{0}\right)\right)
\end{aligned}
$$

for all $t \geq t_{0}$. By using inequalities in (3.3) we get

$$
\begin{aligned}
\left\|\phi\left(t, t_{0}, x_{0}\right)\right\| \leq K e^{\beta\left(t-t_{0}\right)}\left\|x_{0}\right\|+\int_{t_{0}}^{t} K e^{\beta(t-s)} l\left\|\phi\left(s, t_{0}, x_{0}\right)\right\| d s & \\
& +\sum_{t_{0} \leq \theta_{i}<t} K e^{\beta\left(t-\theta_{i}\right)} l\left\|\phi\left(\theta_{i}, t_{0}, x_{0}\right)\right\|
\end{aligned}
$$

for all $t \geq t_{0}$ is fulfilled. The last expression can be rewritten as

$$
\begin{aligned}
e^{-\beta t}\left\|\phi\left(t, t_{0}, x_{0}\right)\right\| \leq K e^{-\beta t_{0}}\left\|x_{0}\right\|+\int_{t_{0}}^{t} K l e^{-\beta s} & \left\|\phi\left(s, t_{0}, x_{0}\right)\right\| d s \\
& +\sum_{t_{0} \leq \theta_{i}<t} K l e^{-\beta \theta_{i}}\left\|\phi\left(\theta_{i}, t_{0}, x_{0}\right)\right\|
\end{aligned}
$$

for all $t \geq t_{0}$. Hence, by Gronwall-Bellman lemma for piecewise continuous functions $([4,19])$ it follows that

$$
\left\|\phi\left(t, t_{0}, x_{0}\right)\right\| \leq K e^{(\beta+K l)\left(t-t_{0}\right)}(1+K l)^{i\left[t_{0}, t\right)}\left\|x_{0}\right\| \text { for all } t \geq t_{0} .
$$

By means of of the inequality $\theta_{i+1}-\theta_{i} \geq \underline{\theta}$ one can see that

$$
\left\|\phi\left(t, t_{0}, x_{0}\right)\right\| \leq K e^{\left(\beta+K l+\frac{1}{\underline{\theta}} \ln (1+K l)\right)\left(t-t_{0}\right)}\left\|x_{0}\right\| \text { for all } t \geq t_{0} .
$$

If $l$ is small enough that

$$
\beta+K l+\frac{1}{\underline{\theta}} \ln (1+K l)<0 \text { for } \beta<0,
$$

then the required result follows by choosing $\delta=K h$.

Theorem 2. If there exist $\beta>0, K \geq 1$ and $\delta>0$ such that

$$
\|\Phi(t, s)\| \leq K e^{\beta(t-s)} \text { for all } t \leq s,
$$

and the functions $F(t, x)$ and $I_{i}(x)$ satisfy the inequalities

$$
\|F(t, x)\| \leq l\|x\|, \quad\left\|I_{i}(x)\right\| \leq l\|x\|
$$

for all $t \in I, i \in \mathbb{A}$ and $\|x\|<h, h>0$. Then,

$$
\left\|\phi\left(t, t_{0}, x_{0}\right)\right\| \leq \delta e^{\left(\beta-K l+\frac{1}{\underline{\theta}} \ln (1-K l)\right)\left(t-t_{0}\right)} \text { for all } t, t_{0} \in I \text { with } t \leq t_{0},
$$


i.e., for sufficiently small values of $l$, the trivial solution of (3.1) is all-time (past, future, respectively) repulsive.

Proof. An equivalent integral equation of the system (3.1) can be written as $[4,19]$ :

$$
\begin{aligned}
\phi\left(t, t_{0}, x_{0}\right)=\Phi\left(t, t_{0}\right) x_{0}+\int_{t_{0}}^{t} \Phi(t, s) F\left(s, \phi\left(s, t_{0}, x_{0}\right)\right) d s & \\
& -\sum_{t \leq \theta_{i}<t_{0}} \Phi\left(t, \theta_{i}\right) I_{i}\left(\phi\left(\theta_{i}, t_{0}, x_{0}\right)\right)
\end{aligned}
$$

for all $t \leq t_{0}$. By using inequalities in (3.3) we get

$$
\begin{aligned}
\left\|\phi\left(t, t_{0}, x_{0}\right)\right\| \leq K e^{\beta\left(t-t_{0}\right)}\left\|x_{0}\right\| & +\int_{t_{0}}^{t} K e^{\beta(t-s)} l\left\|\phi\left(s, t_{0}, x_{0}\right)\right\| d s \\
& +\sum_{t \leq \theta_{i}<t_{0}} K e^{\beta\left(t-\theta_{i}\right)} l\left\|\phi\left(\theta_{i}, t_{0}, x_{0}\right)\right\|
\end{aligned}
$$

for all $t \leq t_{0}$ is fulfilled. The last expression can be rewritten as

$$
\begin{aligned}
e^{-\beta t}\left\|\phi\left(t, t_{0}, x_{0}\right)\right\| \leq K e^{-\beta t_{0}}\left\|x_{0}\right\|+\int_{t_{0}}^{t} K l e^{-\beta s} & \left\|\phi\left(s, t_{0}, x_{0}\right)\right\| d s \\
& +\sum_{t \leq \theta_{i}<t_{0}} K l e^{-\beta \theta_{i}}\left\|\phi\left(\theta_{i}, t_{0}, x_{0}\right)\right\|
\end{aligned}
$$

for all $t \leq t_{0}$. Gronwall-Bellman lemma for piecewise continuous functions ([4,19]) can be applied since $l$ can be chosen that $K l<1$. Thus,

$$
\left\|\phi\left(t, t_{0}, x_{0}\right)\right\| \leq K e^{(\beta-K l)\left(t-t_{0}\right)}(1-K l)^{-i\left[t, t_{0}\right)}\left\|x_{0}\right\| \text { for all } t \leq t_{0} .
$$

By means of of the inequality $\theta_{i+1}-\theta_{i} \geq \underline{\theta}$ one can see that

$$
\left\|\phi\left(t, t_{0}, x_{0}\right)\right\| \leq K e^{\left(\beta-K l+\frac{1}{\underline{\underline{\theta}}} \ln (1-K l)\right)\left(t-t_{0}\right)}\left\|x_{0}\right\| \text { for all } t \leq t_{0} .
$$

If $l$ is small enough that

$$
\beta-K l+\frac{1}{\underline{\theta}} \ln (1-K l)>0 \text { for } \beta>0,
$$

then the required results follow if we choose $\delta=K h$ since $\left\|x_{0}\right\|<h$.

\section{THE TRANSCRITICAL BIFURCATION}

In this section we study impulsive analogue of the nonautonomous transcritical bifurcation. Let $x_{-}<0<x_{+}$and $\alpha_{-}<\alpha_{+}$be real numbers and $I$ be interval of the 
form $\mathbb{R}, \mathbb{R}_{k}^{-}$or $\mathbb{R}_{k}^{+}$, respectively. Consider the system

$$
\begin{aligned}
& \dot{x}=a(t, \alpha) x+b(t, \alpha) x^{2}+r(t, x, \alpha), \\
& \left.\Delta x\right|_{t=\theta_{i}}=c_{i}(\alpha) x+d_{i}(\alpha) x^{2}+e_{i}(x, \alpha),
\end{aligned}
$$

with piecewise continuous functions $a: I \times\left(\alpha_{-}, \alpha_{+}\right) \rightarrow \mathbb{R}, b: I \times\left(\alpha_{-}, \alpha_{+}\right) \rightarrow \mathbb{R}$ and $r: I \times\left(x_{-}, x_{+}\right) \times\left(\alpha_{-}, \alpha_{+}\right) \rightarrow \mathbb{R}$ satisfying $r(t, 0, \alpha)=0 . c: \mathbb{A} \times\left(\alpha_{-}, \alpha_{+}\right) \rightarrow$ $\mathbb{R}, d: \mathbb{A} \times\left(\alpha_{-}, \alpha_{+}\right) \rightarrow \mathbb{R}$ and $e: \mathbb{A} \times\left(x_{-}, x_{+}\right) \times\left(\alpha_{-}, \alpha_{+}\right) \rightarrow \mathbb{R}$ with $c_{i}(\alpha) \neq-1$ and $e_{i}(0, \alpha)=0$. An infinite sequence $\theta_{i}$ satisfies $\left|\theta_{i}\right| \rightarrow \infty$ as $|i| \rightarrow \infty$. It is assumed that there exist positive constants $\underline{\theta}$ and $\bar{\theta}$ such that $\underline{\theta} \leq \theta_{i+1}-\theta_{i} \leq \bar{\theta}$. Let $\Phi_{\alpha}(t, s)$ be the fundamental matrix of the linear system

$$
\begin{aligned}
& \dot{x}=a(t, \alpha) x, \\
& \left.\Delta x\right|_{t=\theta_{i}}=c_{i}(\alpha) x .
\end{aligned}
$$

Assume that there exists $\alpha_{0} \in\left(\alpha_{-}, \alpha_{+}\right)$such that are two functions $\beta_{1}, \beta_{2}:\left(\alpha_{-}, \alpha_{+}\right) \rightarrow$ $\mathbb{R}$ which are either both monotone increasing or both monotone decreasing and $K \geq 1$ satisfying $\lim _{\alpha \rightarrow \alpha_{0}} \beta_{1}(\alpha)=\lim _{\alpha \rightarrow \alpha_{0}} \beta_{2}(\alpha)=0$ and

$$
\begin{array}{ll}
\Phi_{\alpha}(t, s) \leq K e^{\beta_{1}(\alpha)(t-s)} & \text { for all } \alpha \in\left(\alpha_{-}, \alpha_{+}\right) \text {and } t, s \in I \text { with } t \geq s, \\
\Phi_{\alpha}(t, s) \leq K e^{\beta_{2}(\alpha)(t-s)} & \text { for all } \alpha \in\left(\alpha_{-}, \alpha_{+}\right) \text {and } t, s \in I \text { with } t \leq s .
\end{array}
$$

The quadratic terms either fulfill

$$
\begin{aligned}
& 0<\liminf _{\alpha \rightarrow \alpha_{0}} \inf _{t \in I} b(t, \alpha) \leq \limsup _{\alpha \rightarrow \alpha_{0}} \sup _{t \in I} b(t, \alpha)<\infty, \\
& 0<\liminf _{\alpha \rightarrow \alpha_{0}} \inf _{i \in \mathbb{A}} d_{i}(\alpha) \leq \limsup _{\alpha \rightarrow \alpha_{0}} \sup _{i \in \mathbb{A}} d_{i}(\alpha)<\infty
\end{aligned}
$$

or

$$
\begin{aligned}
& -\infty<\liminf _{\alpha \rightarrow \alpha_{0}} \inf _{t \in I} b(t, \alpha) \leq \limsup _{\alpha \rightarrow \alpha_{0}} \sup _{t \in I} b(t, \alpha)<0, \\
& -\infty<\liminf _{\alpha \rightarrow \alpha_{0}} \inf _{i \in \mathbb{A}} d_{i}(\alpha) \leq \limsup _{\alpha \rightarrow \alpha_{0}} \sup _{i \in \mathbb{A}} d_{i}(\alpha)<0,
\end{aligned}
$$

and the remainders satisfy

$$
\begin{aligned}
& \lim _{x \rightarrow 0} \sup _{\alpha \in\left(\alpha_{0}-|x|, \alpha_{0}+|x|\right)} \sup _{t \in I} \frac{|r(t, x, \alpha)|}{|x|^{2}}=0, \\
& \lim _{x \rightarrow 0} \sup _{\alpha \in\left(\alpha_{0}-|x|, \alpha_{0}+|x|\right)} \sup _{i \in \mathbb{A}} \frac{\left|e_{i}(x, \alpha)\right|}{|x|^{2}}=0
\end{aligned}
$$

and there exists sufficiently small $l>0$ such that

$$
|r(t, x, \alpha)|<l|x|,\left|e_{i}(x, \alpha)\right|<l|x|,
$$

for all $\alpha \in\left(\alpha_{-}, \alpha_{+}\right), t \in I, i \in \mathbb{A}$ and $x \in\left(x_{-}, x_{+}\right)$. 
Theorem 3. Assume that above conditions hold for the system (4.1). Then there exist $\widehat{\alpha}_{-}<0<\widehat{\alpha}_{+}$such that

- If the functions $\beta_{1}$ and $\beta_{2}$ are monotone increasing, the trivial solution is all-time (past, future, respectively) attractive for $\alpha \in\left(\widehat{\alpha}_{-}, \alpha_{0}\right)$ and all-time repulsive for $\alpha \in\left(\alpha_{0}, \widehat{\alpha}_{+}\right)$. The system (4.1) admits an all-time (past, future, respectively) bifurcation, since the corresponding radii of all-time (past, future, respectively) attraction and repulsion satisfy

$$
\lim _{\alpha \nearrow \alpha_{0}} \mathcal{A}_{0}^{\alpha}=0 \text { and } \lim _{\alpha \searrow \alpha_{0}} \mathcal{R}_{0}^{\alpha}=0 .
$$

- If the functions $\beta_{1}$ and $\beta_{2}$ are monotone decreasing, the trivial solution is all-time (past, future, respectively) repulsive for $\alpha \in\left(\widehat{\alpha}_{-}, \alpha_{0}\right)$ and all-time attractive for $\alpha \in\left(\alpha_{0}, \widehat{\alpha}_{+}\right)$. The system (4.1) admits an all-time (past, future, respectively) bifurcation, since the corresponding radii of all-time (past, future, respectively) attraction and repulsion satisfy

$$
\lim _{\alpha \nearrow \alpha_{0}} \mathcal{R}_{0}^{\alpha}=0 \text { and } \lim _{\alpha \searrow \alpha_{0}} \mathcal{A}_{0}^{\alpha}=0 .
$$

Proof. We prove the first part since the second part can be proven in a similar manner. Let $\phi_{\alpha}$ be the general solution of the system (4.1). We may consider the case (4.3) since the functions $\beta_{1}$ and $\beta_{2}$ are monotone increasing. Choose $\widehat{\alpha}_{-}<\alpha_{0}<\widehat{\alpha}_{+}$ such that

$$
\begin{aligned}
& 0<\inf _{\alpha \in\left(\widehat{\alpha}_{-}, \widehat{\alpha}_{+}\right), t \in I} b(t, \alpha) \leq \sup _{\alpha \in\left(\widehat{\alpha}_{-}, \widehat{\alpha}_{+}\right), t \in I} b(t, \alpha)<\infty, \\
& 0<\inf _{\alpha \in\left(\widehat{\alpha}_{-}, \widehat{\alpha}_{+}\right), i \in \mathbb{A}} d_{i}(\alpha) \leq \sup _{\alpha \in\left(\widehat{\alpha}_{-}, \widehat{\alpha}_{+}\right), i \in \mathbb{A}} \\
& d_{i}(\alpha)<\infty .
\end{aligned}
$$

By means of (4.6) and (4.9) one can see that Theorem 1 and Theorem 2 can be applied. Thus, we get attractivity and repulsivity of the trivial solution as it was required to show in the theorem. To prove relations (4.7) and (4.8) we assume to the contrary that

$$
\gamma:=\limsup _{\alpha \nearrow \alpha_{0}} \mathcal{A}_{0}^{\alpha}>0
$$

By means of (4.5) and (4.9) one can show that there exist $\widetilde{\alpha} \in\left(\widehat{\alpha}_{-}, \alpha_{0}\right), x_{0} \in(0, \gamma)$ and $L \in\left(0, \frac{x_{0}}{4 K}\right)$ such that

$$
b(t, \alpha) x^{2}+r(t, x, \alpha)>L \text { and } d_{i}(\alpha) x^{2}+e_{i}(x, \alpha)>L
$$

for all $t \in I, i \in \mathbb{A}, \alpha \in\left(\widetilde{\alpha}_{-}, \alpha_{0}\right)$ and $x_{0} \in\left[\frac{x_{0}}{2 K^{2}}, x_{0}\right]$. Next, fix $\widehat{\alpha} \in\left(\widetilde{\alpha}_{-}, \alpha_{0}\right)$ such that $\mathcal{A}_{0}^{\widehat{\alpha}}>x_{0}$ and $\beta_{2}(\widehat{\alpha}) \geq \beta:=-\frac{2 K L}{x_{0}}>-\frac{1}{2}$ so that $\phi_{\widehat{\alpha}}\left(t, t_{0}, x_{0}\right)$ is attracted to the origin. Denote $\psi(t)=\phi_{\widehat{\alpha}}\left(t, t_{0}, x_{0}\right)$. Then, there exists $t_{2} \in I, t_{2}>t_{0}$, such that $\psi\left(t_{2}\right) \leq \frac{x_{0}}{2 K^{2}}$. 
Choose minimal $t_{2}$ with this property, i.e., $\psi\left(t_{2}\right)>\frac{x_{0}}{2 K^{2}}$ for all $t \in\left[t_{0}, t_{2}\right)$. Moreover, choose $t_{1} \in\left[t_{0}, t_{2}\right)$ such that

$$
\psi\left(t_{1}\right)=\frac{x_{0}}{2 K} \text { and } \psi(t) \in\left(\frac{x_{0}}{2 K^{2}}, x_{0}\right] \text { for all } t \in\left[t_{1}, t_{2}\right) .
$$

We write integral equation of the system (4.1) at $t_{2}$ for fixed $\widehat{\alpha}$ which start at point $t_{1}$.

$$
\begin{gathered}
\psi\left(t_{2}\right)=\Phi_{\widehat{\alpha}}\left(t_{2}, t_{1}\right) \psi\left(t_{1}\right)+\int_{t_{1}}^{t_{2}} \Phi_{\widehat{\alpha}}\left(t_{2}, s\right)\left(b(s, \widehat{\alpha})(\psi(s))^{2}+r(s, \psi(s), \widehat{\alpha})\right) d s \\
+\sum_{t_{1} \leq \theta_{i}<t_{2}} \Phi_{\widehat{\alpha}}\left(t_{2}, \theta_{i}\right)\left(d_{i}(\widehat{\alpha})\left(\psi\left(\theta_{i}\right)\right)^{2}+e_{i}\left(\psi\left(\theta_{i}\right), \widehat{\alpha}\right)\right) \\
>\frac{x_{0}}{2 K^{2}} e^{\beta\left(t_{2}-t_{1}\right)}+\frac{L}{K} \int_{t_{1}}^{t_{2}} e^{\beta\left(t_{2}-s\right)} d s \\
=e^{\beta\left(t_{2}-t_{1}\right)}\left(\frac{x_{0}}{2 K^{2}}+\frac{L}{K \beta}\right)-\frac{L}{K \beta}=\frac{x_{0}}{2 K^{2}}
\end{gathered}
$$

which is a contradiction and proves that $\lim _{\alpha \nearrow \alpha_{0}} \mathcal{A}_{0}^{\alpha}=0$. Analogously, one can show that $\lim _{\alpha \searrow \alpha_{0}} \mathcal{R}_{0}^{\alpha}=0$.

\section{THE PITCHFORK BIFURCATION}

In this section we study impulsive analogue of the nonautonomous pitchfork bifurcation. Let $x_{-}<0<x_{+}$and $\alpha_{-}<\alpha_{+}$be real numbers and $I$ be interval of the form $\mathbb{R}, \mathbb{R}_{k}^{-}$or $\mathbb{R}_{k}^{+}$, respectively. Consider the system

$$
\begin{aligned}
& \dot{x}=a(t, \alpha) x+b(t, \alpha) x^{3}+r(t, x, \alpha), \\
& \left.\Delta x\right|_{t=\theta_{i}}=c_{i}(\alpha) x+d_{i}(\alpha) x^{3}+e_{i}(x, \alpha),
\end{aligned}
$$

with piecewise continuous functions $a: I \times\left(\alpha_{-}, \alpha_{+}\right) \rightarrow \mathbb{R}, b: I \times\left(\alpha_{-}, \alpha_{+}\right) \rightarrow \mathbb{R}$ and $r: I \times\left(x_{-}, x_{+}\right) \times\left(\alpha_{-}, \alpha_{+}\right) \rightarrow \mathbb{R}$ satisfying $r(t, 0, \alpha)=0 . c: \mathbb{A} \times\left(\alpha_{-}, \alpha_{+}\right) \rightarrow$ $\mathbb{R}, d: \mathbb{A} \times\left(\alpha_{-}, \alpha_{+}\right) \rightarrow \mathbb{R}$ and $e: \mathbb{A} \times\left(x_{-}, x_{+}\right) \times\left(\alpha_{-}, \alpha_{+}\right) \rightarrow \mathbb{R}$ with $c_{i}(\alpha) \neq-1$ and $e_{i}(0, \alpha)=0$. An infinite sequence $\theta_{i}$ satisfies $\left|\theta_{i}\right| \rightarrow \infty$ as $|i| \rightarrow \infty$. It is assumed that there exist positive constants $\underline{\theta}$ and $\bar{\theta}$ such that $\underline{\theta} \leq \theta_{i+1}-\theta_{i} \leq \bar{\theta}$. Let $\Phi_{\alpha}(t, s)$ be the fundamental matrix of the linear system

$$
\begin{aligned}
& \dot{x}=a(t, \alpha) x, \\
& \left.\Delta x\right|_{t=\theta_{i}}=c_{i}(\alpha) x .
\end{aligned}
$$

Assume that there exists $\alpha_{0} \in\left(\alpha_{-}, \alpha_{+}\right)$such that are two functions $\beta_{1}, \beta_{2}:\left(\alpha_{-}, \alpha_{+}\right) \rightarrow$ $\mathbb{R}$ which are either both monotone increasing or both monotone decreasing and $K \geq 1$ satisfying $\lim _{\alpha \rightarrow \alpha_{0}} \beta_{1}(\alpha)=\lim _{\alpha \rightarrow \alpha_{0}} \beta_{2}(\alpha)=0$ and

$$
\begin{aligned}
& \Phi_{\alpha}(t, s) \leq K e^{\beta_{1}(\alpha)(t-s)} \text { for all } \alpha \in\left(\alpha_{-}, \alpha_{+}\right) \text {and } t, s \in I \text { with } t \geq s, \\
& \Phi_{\alpha}(t, s) \leq K e^{\beta_{2}(\alpha)(t-s)} \text { for all } \alpha \in\left(\alpha_{-}, \alpha_{+}\right) \text {and } t, s \in I \text { with } t \leq s .
\end{aligned}
$$


The quadratic terms either fulfills

$$
\begin{aligned}
& 0<\liminf _{\alpha \rightarrow \alpha_{0}} \inf _{t \in I} b(t, \alpha) \leq \limsup _{\alpha \rightarrow \alpha_{0}} \sup _{t \in I} b(t, \alpha)<\infty, \\
& 0<\liminf _{\alpha \rightarrow \alpha_{0}} \inf _{i \in \mathbb{A}} d_{i}(\alpha) \leq \limsup _{\alpha \rightarrow \alpha_{0}} \sup _{i \in \mathbb{A}} d_{i}(\alpha)<\infty
\end{aligned}
$$

or

$$
\begin{aligned}
& -\infty<\liminf _{\alpha \rightarrow \alpha_{0}} \inf _{t \in I} b(t, \alpha) \leq \limsup _{\alpha \rightarrow \alpha_{0}} \sup _{t \in I} b(t, \alpha)<0, \\
& -\infty<\liminf _{\alpha \rightarrow \alpha_{0}} \inf _{i \in \mathbb{A}} d_{i}(\alpha) \leq \limsup _{\alpha \rightarrow \alpha_{0}} \sup _{i \in \mathbb{A}} d_{i}(\alpha)<0,
\end{aligned}
$$

and the remainders satisfy

$$
\begin{aligned}
& \lim _{x \rightarrow 0} \sup _{\alpha \in\left(\alpha_{0}-x^{2}, \alpha_{0}+x^{2}\right)} \sup _{t \in I} \frac{|r(t, x, \alpha)|}{|x|^{3}}=0, \\
& \lim _{x \rightarrow 0} \sup _{\alpha \in\left(\alpha_{0}-x^{2}, \alpha_{0}+x^{2}\right)} \sup _{i \in \mathbb{A}} \frac{\left|e_{i}(x, \alpha)\right|}{|x|^{3}}=0
\end{aligned}
$$

and there exists sufficiently small $l>0$ such that

$$
|r(t, x, \alpha)|<l|x|,\left|e_{i}(x, \alpha)\right|<l|x|,
$$

for all $\alpha \in\left(\alpha_{-}, \alpha_{+}\right), t \in I, i \in \mathbb{A}$ and $x \in\left(x_{-}, x_{+}\right)$.

Theorem 4. Assume that above conditions hold for the system (5.1). Then there exist $\widehat{\alpha}_{-}<0<\widehat{\alpha}_{+}$such that

- If the functions $\beta_{1}$ and $\beta_{2}$ are monotone increasing, the trivial solution is all-time (past, future, respectively) attractive for $\alpha \in\left(\widehat{\alpha}_{-}, \alpha_{0}\right)$ and all-time repulsive for $\alpha \in\left(\alpha_{0}, \widehat{\alpha}_{+}\right)$. The system (4.1) admits an all-time (past, future, respectively) bifurcation, since the corresponding radii of all-time (past, future, respectively) attraction and repulsion satisfy

$$
\lim _{\alpha \nearrow \alpha_{0}} \mathcal{A}_{0}^{\alpha}=0 \text { and } \lim _{\alpha \searrow \alpha_{0}} \mathcal{R}_{0}^{\alpha}=0 .
$$

- If the functions $\beta_{1}$ and $\beta_{2}$ are monotone decreasing, the trivial solution is all-time (past, future, respectively) repulsive for $\alpha \in\left(\widehat{\alpha}_{-}, \alpha_{0}\right)$ and all-time attractive for $\alpha \in\left(\alpha_{0}, \widehat{\alpha}_{+}\right)$. The system (5.1) admits an all-time (past, future, respectively) bifurcation, since the corresponding radii of all-time (past, future, respectively) attraction and repulsion satisfy

$$
\lim _{\alpha \nearrow \alpha_{0}} \mathcal{R}_{0}^{\alpha}=0 \text { and } \lim _{\alpha \searrow \alpha_{0}} \mathcal{A}_{0}^{\alpha}=0 .
$$

The proof of the theorem is similar to that of Theorem 3 . 


\section{REFERENCES}

[1] M. Akhmet, Nonlinear hybrid continuous/discrete-time models. Atlantis Press, 2011.

[2] M. U. Akhmet, "Perturbations and Hopf bifurcation of the planar discontinuous dynamical system," Nonlinear Anal., Theory Methods Appl., Ser. A, Theory Methods, vol. 60, no. 1, pp. 163-178, 2005.

[3] M. U. Akhmet and M. A. Tleubergenova, "Asymptotic equivalence of the quasilinear impulsive differential equation and the linear ordinary differential equation," Miskolc Math. Notes, vol. 8, no. 2, pp. 117-121, 2007.

[4] M. Akhmet, Principles of discontinuous dynamical systems. Berlin: Springer, 2010.

[5] D. D. Bainov and P. S. Simeonov, Impulsive differential equations. Asymptotic properties of the solutions, ser. Series on Advances in Mathematics for Applied Sciences. Singapore: World Scientific, 1995, vol. 28.

[6] M. A. Bilozerowa and V. M. Evtukhov, "Asymptotic representations of solutions of the differential equation $y^{(n)}=\alpha_{0} p(t) \prod_{i=0}^{n-1} \varphi_{i}\left(y^{(i)}\right)$," Math. Notes, Miskolc, vol. 13, no. 2, pp. 249-270, 2012.

[7] D. N. Cheban, P. E. Kloeden, and B. Schmalfuß, "Pullback attractors in dissipative nonautonomous differential equations under discretization," J. Dyn. Differ. Equations, vol. 13, no. 1, pp. 185-213, 2001.

[8] J. Guckenheimer and R. F. Williams, "Structural stability of Lorenz attractors," Publ. Math., Inst. Hautes Étud. Sci., vol. 50, pp. 59-72, 1979.

[9] T. Hajba and M. Kovács, "Solution of the convex programming problem via second order differential equation system," Math. Notes, Miskolc, vol. 13, no. 1, pp. 23-37, 2012.

[10] P. E. Kloeden, "Pitchfork and transcritical bifurcations in systems with homogeneous nonlinearities and an almost periodic time coefficient," Commun. Pure Appl. Anal., vol. 3, no. 2, pp. 161-173, 2004.

[11] P. E. Kloeden and M. Rasmussen, Nonautonomous dynamical systems, ser. Mathematical Surveys and Monographs. Providence, RI: American Mathematical Society (AMS), 2011, vol. 176.

[12] V. Lakshmikantham, D. D. Bainov, and P. S. Simeonov, Theory of impulsive differential equations, ser. Series in Modern Applied Mathematics. Singapore: World Scientific, 1989, vol. 6.

[13] J. A. Langa, J. C. Robinson, and A. Suárez, "Bifurcations in non-autonomous scalar equations," J. Differ. Equations, vol. 221, no. 1, pp. 1-35, 2006.

[14] J. A. Langa, J. C. Robinson, and A. Suárez, "Stability, instability, and bifurcation phenomena in non-autonomous differential equations," Nonlinearity, vol. 15, no. 3, pp. 887-903, 2002.

[15] P. Li, J. Cao, and Z. Wang, "Robust impulsive synchronization of coupled delayed neural networks with uncertainties," Physica A, vol. 373, pp. 261-272, 2007.

[16] M. Rasmussen, Attractivity and bifurcation for nonautonomous dynamical systems, ser. Lecture Notes in Mathematics. Berlin: Springer, 2007, vol. 1907.

[17] M. Rasmussen, "Nonautonomous bifurcation patterns for one-dimensional differential equations," J. Differ. Equations, vol. 234, no. 1, pp. 267-288, 2007.

[18] M. Ronto and A. M. Samoilenko, Numerical-analytic methods in the theory of boundary-value problems. Singapore: World Scientific, 2000.

[19] A. M. Samoilenko and N. A. Perestyuk, Impulsive differential equations. Transl. from the Russian by Yury Chapovsky, ser. World Scientific Series on Nonlinear Science. Series A. Singapore: World Scientific, 1995, vol. 14.

[20] G. Zhang, Z. Liu, and Z. Ma, "Synchronization of complex dynamical networks via impulsive control," Chaos, vol. 17, no. 4, pp. 043 126, 9, 2007.

[21] J. Zhou, L. Xiang, and Z. Liu, "Synchronization in complex delayed dynamical networks with impulsive effects," Physica A, vol. 384, pp. 684-692, 2007. 
Authors' addresses

M. U. Akhmet

Middle East Technical University, Department of Mathematics, 06531 Ankara, Turkey

E-mail address: marat@metu.edu.t

A. Kashkynbayev

Middle East Technical University, Department of Mathematics, 06531 Ankara, Turkey

E-mail address: ardaky@gmail.com 